\title{
Timing divided attention
}

\author{
Hinze Hogendoorn \\ Utrecht University, Utrecht, The Netherlands \\ and University College London, London, England \\ Thomas A. CaRlson \\ Utrecht University, Utrecht, The Netherlands \\ and University of Maryland, College Park, Maryland \\ Rufin VanRullen \\ Université Paul Sabatier, Toulouse, France \\ AND \\ Frans A. J. Verstraten \\ Utrecht University, Utrecht, The Netherlands
}

\begin{abstract}
Visual attention can be divided over multiple objects or locations. However, there is no single theoretical framework within which the effects of dividing attention can be interpreted. In order to develop such a model, here we manipulated the stage of visual processing at which attention was divided, while simultaneously probing the costs of dividing attention on two dimensions. We show that dividing attention incurs dissociable time and precision costs, which depend on whether attention is divided during monitoring or during access. Dividing attention during monitoring resulted in progressively delayed access to attended locations as additional locations were monitored, as well as a one-off precision cost. When dividing attention during access, time costs were systematically lower at one of the accessed locations than at the other, indicating that divided attention during access, in fact, involves rapid sequential allocation of undivided attention. We propose a model in which divided attention is understood as the simultaneous parallel preparation and subsequent sequential execution of multiple shifts of undivided attention. This interpretation has the potential to bring together diverse findings from both the divided-attention and saccade preparation literature and provides a framework within which to integrate the broad spectrum of divided-attention methodologies.
\end{abstract}

Attention enhances the processing of selected visual information. A fundamental capacity of visual attention is that it can be concurrently allocated to multiple objects or locations (e.g., Castiello \& Umiltà, 1992; Kramer \& Hahn, 1995; McMains \& Somers, 2004). However, the mechanism(s) underlying this ability have been the topic of substantial debate. Among the contested aspects are very fundamental questions, including the maximum number of attentional foci that can be maintained (e.g., Alvarez \& Cavanagh, 2005) and the limits on their spatial resolution (e.g., Gobell, Tseng, \& Sperling, 2004; Intriligator \& Cavanagh, 2001), the degree to which these foci are independent (e.g., Yantis, 1992), and the flexibility with which attention can be allocated to the different foci (e.g., Castiello \& Umiltà, 1992; McCormick, Klein, \& Johnston, 1998). Furthermore, it remains disputed whether attention is divided over locations, objects, or features (e.g., Bichot, Cave, \& Pashler, 1999; Scholl, Pylyshyn, \& Feldman, 2001) and how the spatiotemporal properties of attentional allocation to multiple foci are best understood (e.g., Kramer \& Hahn, 1995; VanRullen, Carlson, \& Cavanagh, 2007; Wolfe, 1998). To date, no single theoretical framework of divided attention has been able to incorporate all the lines of evidence provided by the wide range of experimental paradigms with which it has been studied.

\section{Stages of Visual Processing: \\ Monitoring Versus Access}

Attention can be divided into (at least) two stages of visual processing (Pashler, 1998). In general, accounts of perception and attention distinguish between an early preattentive system in which a limited subset of visual features (such as color, orientation, etc.) are processed rap- 
idly and in parallel and a later capacity-limited attentive system in which additional features (such as the identity of a character or the gender of a face) and feature combinations are analyzed in greater depth (e.g., Palmer, 1995; Treisman \& Gelade, 1980; Shiffrin, Gardner, \& Allmeyer, 1973; Wolfe, 1994). A filter mechanism restricts the information that is transferred from the first to the second stage (e.g., Broadbent, 1958).

Attention might be divided during the first stage, which we will call monitoring: the anticipatory allocation of attention to a specific region of the visual field. In a cuing task, for example, a subset of potential target locations is attended in preparation for the appearance of a target (e.g., Awh \& Pashler, 2000; Castiello \& Umiltà, 1992; Kramer \& Hahn, 1995). Similarly, in a multiple-object-tracking task, observers keep track of a subset of moving objects in order to report their final locations, without yet processing any information about those objects other than their location and direction of motion (Pylyshyn \& Storm, 1988). Importantly, little or no information is transferred to the limited-capacity system at this point. In line with this minimal dependence on limited resources, observers are generally able to track up to four or five independently moving objects with little difficulty (Cavanagh \& Alvarez, 2005). Alternatively, attention might be divided during the second stage, which we will call access: the specific allocation of processing resources to one or more visual stimuli, such that their visual properties become available to consciousness. When attention is divided at this stage by requiring observers to respond to multiple simultaneous targets, multiple stimuli compete for access to limited resources, and marked performance decrements are observed (Duncan, 1980b).

\section{Attentional Mechanisms: Optimization Versus Selection}

The term attention has been used to encompass two potentially distinct visual mechanisms. One of these involves accelerating the processing of incoming visual information - a role Huang and Pashler (2007) termed processing optimization. In cuing paradigms, for example, attention is directed to a specific location in the visual field, and reductions in reaction time are observed for targets presented at that location (e.g., Posner, 1980). The effects of optimization are generally modest, with only small differences in speed between attended and unattended targets. Furthermore, although responses to unattended targets are slower, those targets are, in general, still perceived. Reducing the attentional resources available for optimization at a particular location can therefore be expected to result in a relative processing delay at that location. However, unless the task is severely time limited (e.g., as a result of masking), it would not necessarily result in a reduction in the precision with which visual information is encoded at that location. In terms of optimization, the cost of dividing attention can therefore be expected to primarily take the form of a time cost.

The other mechanism involves the selection of a subset of incoming visual input. This is the role attributed to atten- tion in models of feature binding (e.g., Treisman \& Gelade, 1980), in attentive tracking (Cavanagh, 1992; Pylyshyn \& Storm, 1988), and in explanations of change blindness phenomena (e.g., Rensink, O’Regan, \& Clark, 1997). In this role, attention maintains pointers to locations in the visual field, passing on information from the selected locations while discarding information from other locations (Huang $\&$ Pashler, 2007). Reducing the attentional resources available to carry out selection might cause visual features in a scene to become unbound or bound incorrectly (as in the case of illusory conjunctions; Treisman \& Gelade, 1980) or to be encoded or represented with lower fidelity. Targets might be confused with distractors or simply fail to reach awareness at all (Rensink et al., 1997). Failures of selection are therefore more likely to be evident as perceptual errors than as systematic increases in processing time. Accordingly, in terms of selection, the cost of dividing attention can be expected to manifest as a precision cost.

As Huang and Pashler (2007) noted, failing to make this distinction explicit has the potential to generate confusion. An unattended item is perceived relatively late in a cuing task but is not perceived at all in a change blindness task. We can obviously perceive the relative positions of two spatially separate objects, which requires attention at each location in the selection sense. Nonetheless, the question of whether the presence of attention can be demonstrated (in terms of optimization) at similarly separated locations has seen considerable study (e.g., Awh \& Pashler, 2000; Castiello \& Umiltà, 1992; Kramer \& Hahn, 1995). Importantly, in many experimental paradigms, the two mechanisms are confounded by a speed-accuracy trade-off. Under time-limited conditions, processing delays will result in failures to access the target on time, resulting in reduced accuracy. Conversely, in the absence of such a time limit, selection of a (static) stimulus can be reattempted at a cost to reaction time. In this way, time and precision costs that are, in principle, dissociable and likely to be the result of distinct attentional mechanisms become lost in a general performance measure. Therefore, in order to develop a general model of divided attention, we need an experimental paradigm in which time and precision costs can be independently estimated.

\section{Timing Divided Attention}

The costs and limits of dividing attention will clearly depend on both the level of visual processing at which attention is divided (monitoring or access) and which functional role of attention is under consideration (optimization or selection). Here, we present a series of psychophysical experiments in which we probed the costs of dividing attention both when attention was divided during monitoring and when it was divided during access. In addition, within one paradigm, we simultaneously probed the costs of dividing attention on both the time and precision dimensions. This allowed us to attempt to dissociate the effects of dividing attention (at each stage) on optimization and selection mechanisms, respectively. To do so, we adapted our previously developed clocks paradigm (Carlson, Hogendoorn, \& Verstraten, 2006; Libet, 2004; Wundt, 1883). In this para- 
digm, an observer views an array of running clocks, one of which is briefly cued. At the end of each trial, the observer is asked to report the time he or she perceived in the target clock when it was cued. On the basis of the difference between the reported time and the veridical orientation of the clock hand when it was cued (i.e., the response error), we calculate two dependent measures to estimate the time cost and precision cost of dividing attention.

Time cost. The mean response error in each condition was taken as an estimate of the time cost of attentional access in that condition. The clocks paradigm provides a fairly direct measure of the latency of attentional access, because the measure depends on what the observer perceived (i.e., the state of the stimulus when it was accessed by attention), rather than on when the observer perceived it. As such, it avoids the additional delay caused by cognitive and motor processes that are necessarily involved in speeded overt responses. These may themselves be affected by or interact with attentional manipulations (Duncan, 1980a; Palmer, 1995), thereby confounding estimates of the influence of divided attention on perceptual processes. Although cognitive processes are necessarily involved in the clocks task (to encode, remember, and finally report the position of the clock hand), these are unlikely to bias the mean response error in a given condition. Since this measure probes the latency of attentional access, it measures differences in time cost and, therefore, the optimization mechanism of attention.

Precision cost. The variability of response errors in a given condition was taken as an estimate of spatiotemporal precision. This measure is sensitive not just to variability in when attentional access itself takes place, but also to the quality of the observer's percept. Any source of noise in observer responses, including cognitive factors such as the decay of a memory trace, contributes to this measure. This allows us to quantify to a degree the success or failure of attentional selection. Although failure of attentional selection might have a wide range of effects, the majority of these would result in additional variability in observer responses. The variability of response errors is thus a useful aggregate measure for probing differences in precision cost and, therefore, the selection mechanism of attention.

Here, we adapted the clocks task such that observers were required to divide attention at different levels of processing, in order to compare the costs of dividing attention during monitoring and access simultaneously on two different dimensions. In Experiment 1, we manipulated the subset of clocks that were indicated before the start of the trial, such that the observer was required to monitor between one and six clocks for the appearance of a single cue. In Experiment 2, observers always monitored two clocks, and we instead manipulated whether observers were required to report either one or both clocks. In this way, we manipulated whether attention was divided during access. On the basis of previous work (Carlson et al., 2006), we anticipated additional time costs as observers divided attention to monitor additional clocks. In line with Duncan (1980b), we anticipated that dividing attention to simultaneously access two clocks, rather than one, would result in additional precision costs.

\section{METHOD}

\section{Observers}

Ten observers participated in both experiments: 9 observers naive as to the purpose of the study and 1 of the authors (H.H.). All had normal or corrected-to-normal vision. All the observers gave informed consent prior to participation. Although no preselection was applied, all the observers were right-handed.

\section{Stimuli}

We adapted the clocks paradigm we previously developed to measure the speed of attention (Carlson et al., 2006; Hogendoorn, Carlson, \& Verstraten, 2007; MATLAB code is freely available to download and use). Here, we adapted the paradigm to investigate the costs of dividing attention. In Experiment 1, the stimuli consisted of six running analog clocks (diameter, $3.7^{\circ}$ ), presented on an imaginary circle at $5.7^{\circ}$ from a central fixation point. Each clock featured a single hand revolving clockwise at $1 \mathrm{~Hz}$ (Figure 1, left panel). At cue time, one of the clocks was exogenously cued by flashing its rim from black to bright red. In Experiment 2, the stimuli instead consisted of just two running analog clocks (diameter, $3.7^{\circ}$ ), presented at $3.7^{\circ}$ left and right of a central fixation point. Each clock featured a single hand revolving at $1 \mathrm{~Hz}$, with the left and right clock hands revolving in opposite directions. Which of the two clock hands revolved clockwise was randomized on each trial (Figure 1, right panel).

All the stimuli were displayed on a 21 -in. Silicon Graphics CRT monitor $(100 \mathrm{~Hz}, 1,152 \times 864$ pixel resolution $)$ at a viewing distance of $57 \mathrm{~cm}$, controlled by a PC running MATLAB 6.5 using PsychToolbox 2.54 extensions (Brainard, 1997; Pelli, 1997). The stimuli were presented on a $50 \%$ gray background. Eye movements were monitored for 3 of the 10 observers using a Cambridge Research Systems High Speed Video Eyetracker sampling the observer's right eye at $250 \mathrm{~Hz}$. For these observers, stimuli were displayed on a 21 -in. Mitsubishi Diamondtron CRT monitor $(85 \mathrm{~Hz}, 1,152 \times$ 864 pixel resolution) at a viewing distance of $80 \mathrm{~cm}$.

\section{Procedure}

The observers were briefly familiarized with the stimulus and the task, instructed to maintain central fixation during both experiments, and given several practice trials. In particular, the observers were instructed to report the time that they perceived in the cued clock(s) at the time of the cue(s), and not to attempt to strategically compensate for any perceived delay. The initial positions of the clock hands were randomized. All the observers completed Experiment 1 before completing Experiment 2 .

Experiment 1. In Experiment 1, before each trial, from one to six of a total of six clocks were indicated by short lines extending from the fixation point in the direction of each of the clocks. These served to indicate to the observer which subset of the six clocks to monitor for a cue. This indication was $100 \%$ valid, in that the clock that would be cued on a given trial was always among those indicated. The monitor 1 condition was therefore maximally informative, whereas the monitor 6 condition (in which all clocks were to be monitored) was least informative. The lines were presented for $1 \mathrm{sec}$ and then removed from the screen. One second later, all six running clocks were presented for a total of $3 \mathrm{sec}$. At a random moment between 1 and $2 \mathrm{sec}$ after onset, one of the clocks was cued by changing the color of the rim from black to bright red for $20 \mathrm{msec}$. At the end of the trial, the clocks disappeared, and the observer was presented with a single, centrally presented clock with a random initial hand position, which could be adjusted using the keyboard. The observer's task was to use this clock to indicate the time that he or she perceived on the cued clock when it was cued. All the observers completed 60 trials in each of the six conditions, in random order, resulting in a total of 360 trials. 


\section{Experiment 1}

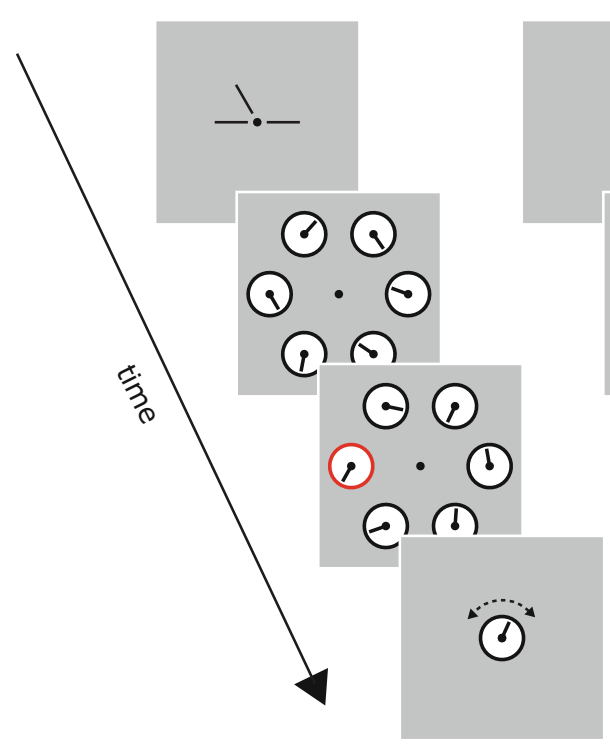

\section{Experiment 2}

Figure 1. Examples of stimuli. In Experiment 1 (left), the stimulus consisted of six clocks. On each trial, a subset of the six clocks was indicated before the trial, such that observers could selectively monitor the subset for the appearance of a cue. One of the indicated clocks was cued during the trial by changing the rim of the clock to red. The observers reported the time on the cued clock. In Experiment 2 (right), the stimulus consisted of two clocks, of which either or both could be cued by changing the rim of the clock to red (a double-cue trial is shown). On single-clock trials, the observers reported only the time on the cued clock; on double-cue trials, the observers reported the time on each of the two clocks.
Experiment 2. In Experiment 2, no clocks were indicated before the trials: Two clocks were presented, and the observers monitored both clocks on all trials. Instead, we manipulated whether one or both of the two clocks were cued. The two running clocks were presented for $3 \mathrm{sec}$. At a random moment between 1 and $2 \mathrm{sec}$ after the start of the trial, either one or both of the clocks were cued by changing the color of the rim from black to bright red for $20 \mathrm{msec}$. At the end of the trial, the observer was presented with either one or two clocks, depending on whether one or both clocks were cued during the trial. These test clocks had been presented in the same positions as the clocks during the trial and could be adjusted using the keyboard. Initial positions of the clock hands were randomized. The observer's task was to use each clock to indicate the perceived time on the appropriate clock when it was cued during the trial. All the observers completed 400 trials in random order ( 80 trials on which the left clock was cued, 80 trials on which the right clock was cued, and 240 trials on which both clocks were cued).

\section{RESULTS}

The difference between the veridical and reported times on the cued clock(s) (i.e., the response error) was calculated on each trial. Since all clock hands revolved at $1 \mathrm{~Hz}$, reported positions up to $180^{\circ}$ ahead of the clock hand were taken as positive errors, and reported positions up to $180^{\circ}$ behind the clock hand were taken as negative errors. Although, in principle, the circular nature of the response could confound large positive and large negative errors, in practice, the observers' response distributions were sufficiently narrow to negate this problem. From this difference, two dependent measures were calculated in each condition: (1) The mean response error within each condition was taken as a measure of the time cost of deploying attention in a particular condition, and (2) the standard deviation of response errors within each condition was taken as a measure of the precision cost.

\section{Experiment 1}

In Experiment 1, we investigated the time and precision costs of dividing attention over multiple possible cue locations. The observers monitored a variable number of clocks for the appearance of a cue. Mean time and precision costs of dividing attention over one to six locations are shown in Figure 2 (left panels). Mean time and precision costs were entered into one-way repeated measures ANOVAs, with number of monitored clocks as the only factor. A significant effect of the number of monitored clocks was observed for both time cost $[F(5)=17.94, p<$ $.001]$ and precision cost $[F(5)=3.12, p=.017]$.

Additional time costs were the observed when observers divided attention in order to monitor multiple locations. Time costs increased gradually with the number of monitored locations and reached an asymptote as observers monitored 5 clocks (Figure 2A). The asymptote of around $100 \mathrm{msec}$ is in good agreement with our previous estimates of the time cost of a single exogenously cued attentional shift (using an otherwise identical method with an array of 10 clocks, all of which were potential targets, we observed mean time costs of $\sim 120 \mathrm{msec}$; Carlson et al., 2006). Mean time costs when one, two, or 
A

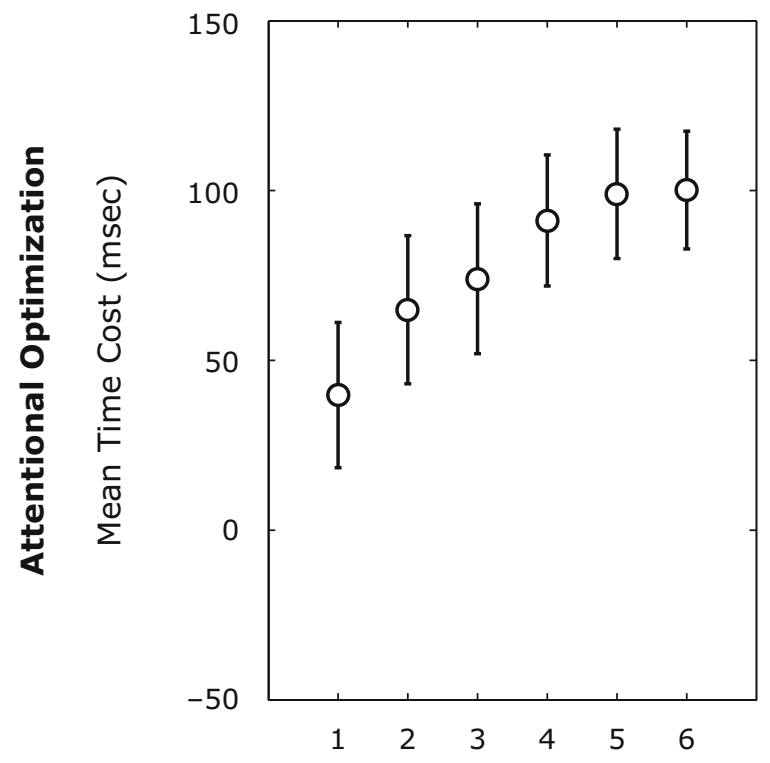

Number of Monitored Clocks
B

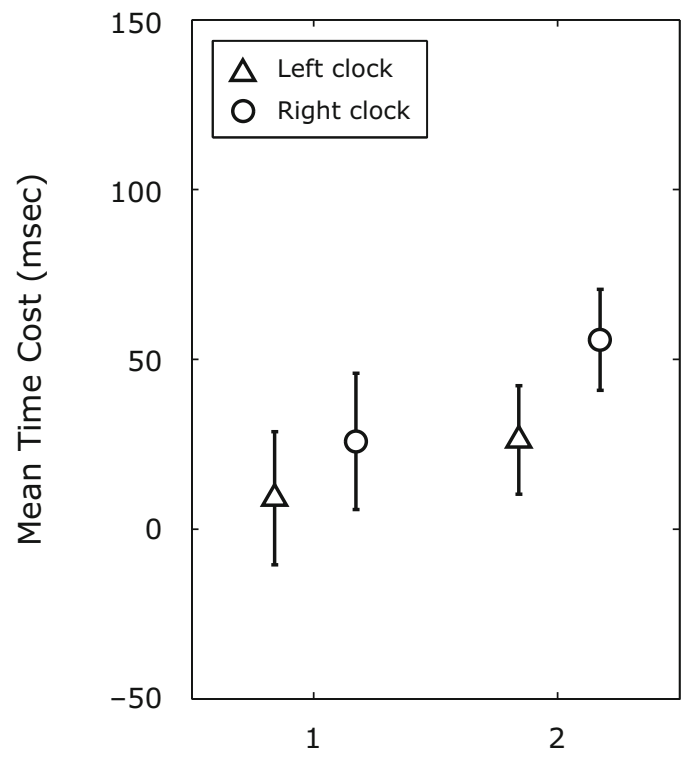

Number of Accessed Clocks
C

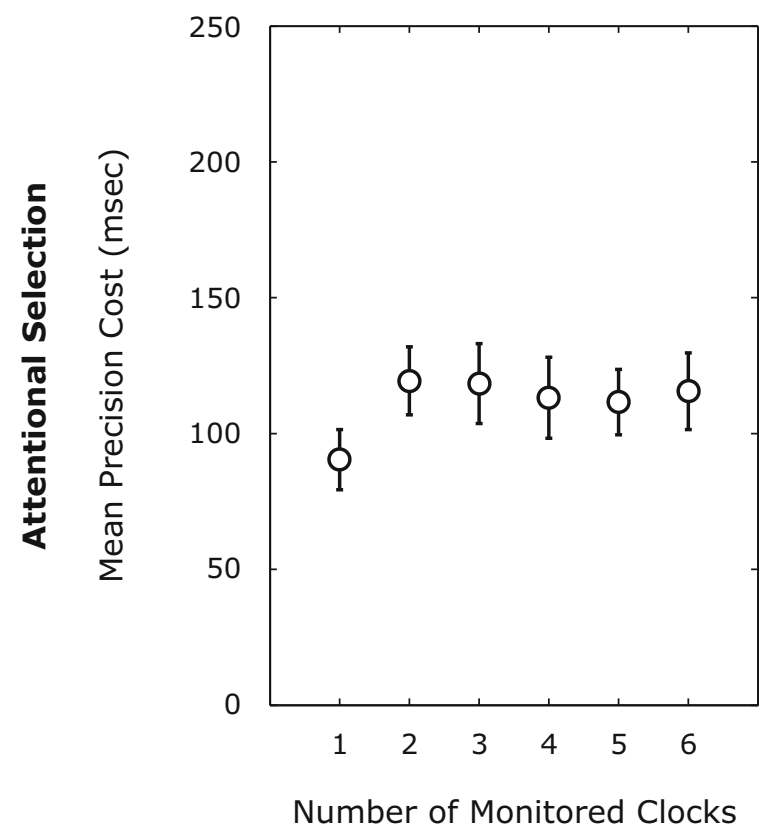

D

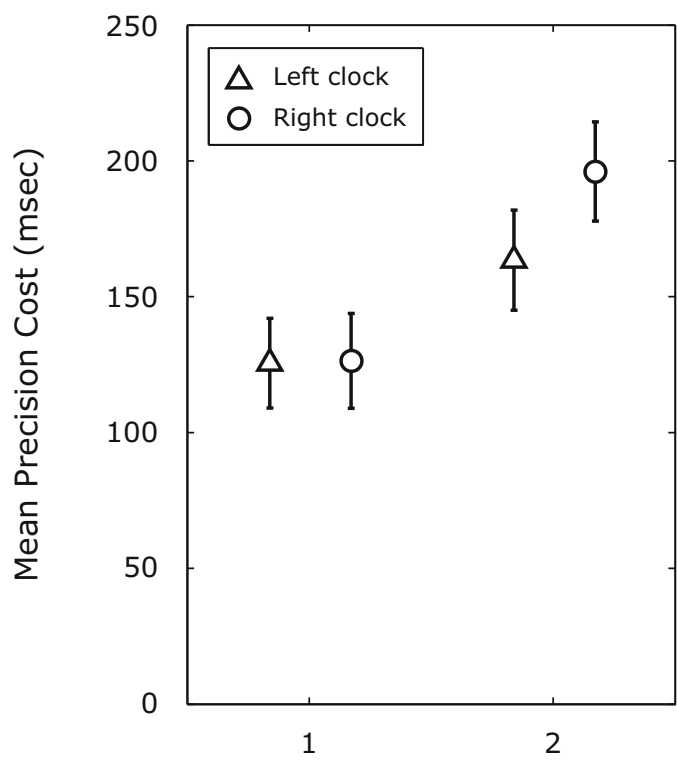

Number of Accessed Clocks

Figure 2. Costs of dividing attention. Time (as mean response error) and precision (as the standard deviation of response errors) were taken as separate measures of attentional optimization and attentional selection, respectively. Panels $\mathrm{A}$ and $\mathrm{C}$ show the time cost and precision cost of dividing attention during monitoring, as a function of the number of monitored clocks (Experiment 1). Time cost progressively increased as observers monitored more clocks (panel A), reaching an asymptote at around five clocks. Conversely, precision costs show a discrete pattern, with low cost when attention is undivided and high cost when attention is divided, irrespective of the number of monitored locations. Panels $B$ and $D$ show time and precision costs, respectively, for dividing attention during access (Experiment 2). Costs are plotted separately for single-cue and double-cue trials and separately for the left and right clocks (triangles and circles, respectively). Double-cue trials show greater time and precision costs than do single-cue trials. In addition, right-clock reports consistently showed greater time and precision costs than did left-clock reports, on both single- and double-cue trials. 
three locations were monitored were significantly lower than when five or six locations were monitored (TukeyKramer corrected paired-sample $t$ tests, all $p \mathrm{~s}<.05$ ). Reductions in time cost (as compared with when attention was divided to monitor all locations) were therefore observed at up to three monitored locations. This reduction in time cost, relative to the asymptote, is the result of attentional resources being available to allow proportionally more rapid access to visual information at the attended locations. Overall, the magnitude of this reduction was roughly proportional to the reciprocal of the number of monitored locations.

Dividing attention during monitoring also resulted in increased costs to precision. However, the pattern of costs was very different, increasing only as attention was divided from one to two locations, and remaining stable as the number of attended locations increased further (Figure $2 \mathrm{C}$ ). This one-off precision cost suggests that monitoring one location is qualitatively different from dividing attention to monitor two or more locations. This result is easily understood in terms of the requirements of attentional selection, given that when the cue appears in the monitor 1 condition, the target clock is the only selected clock. Conversely, in the monitor 2-6 conditions, multiple clocks are selected when the cue appears, and the selection needs to be modified (i.e., reduced to only the target clock), leading to a cost in precision.

Together, the time and precision results suggest that dividing attention over multiple locations during monitoring is achieved by a parallel, rather than a serial, mechanism. In a serial model, in which a single attentional focus switches rapidly between monitored locations, the proportion of trials on which attention happens to be allocated to the cued clock when it is cued decreases with the reciprocal of the number of monitored clocks. A parallel model predicts the same, since the total attentional capacity must be divided over the monitored locations. Both accounts are therefore compatible with our time cost result. However, a serial account would predict a similar gradual reduction in precision as additional clocks are monitored, rather than the one-off cost to precision that we observed. Altogether, the results of Experiment 1 are therefore consistent with the interpretation that optimization effects can be observed in parallel at up to four monitored locations but that, in terms of selection, divided attention is qualitatively different from undivided attention.

\section{Experiment 2}

In Experiment 2, we investigated the time and precision costs of dividing attentional resources during attentional access. The observers monitored two clocks for the appearance of either one or two cues. Time and precision costs of accessing visual information at either one or two locations are shown in Figure 2 (right panels). Mean time and precision costs were entered into $2 \times 2$ repeated measures ANOVAs, with number of clocks selected (one or two) and clock identity (left or right) as factors. A significant main effect of the number of selected clocks was observed on both time $[F(1)=6.39, p=.032]$ and precision $[F(1)=117.26, p<.001]$ costs. Simultaneously accessing both clocks, rather than one, resulted in both additional time and additional precision costs. ${ }^{1}$

In addition, we observed an unexpected main effect of clock identity on both time $[F(1)=14.95, p=.004]$ and precision $[F(1)=5.76, p=.040]$. Time costs were consistently lower for the left clock than for the right clock, irrespective of whether one or both clocks were selected (Figure 2B). Similarly, precision was greater for left-clock responses than for right-clock responses, on both singleand double-cue trials. Although the interaction between number of reported clocks and clock identity was not significant for either time or precision $[F(1)=0.89, p=$ .371 , and $F(1)=3.05, p=.115$, respectively], the precision difference between the left and right clocks was most pronounced when two clocks were reported (Figure 2D).

There was no a priori difference between the two clocks in the stimulus. Instead, this pattern of results suggests that the observers spontaneously chose to prepare for the possibility that visual information would need to be simultaneously selected at two locations by strategically preallocating processing resources to one location before the other. Furthermore, the observers did so consistently from trial to trial, systematically favoring the left clock over the right. As a result, right-clock reports show additional time and precision costs on both single- and double-cue trials. Strikingly, the observers were almost unanimous in adopting this strategy: The left-clock advantage was evident in 8 of the 10 observers individually.

Finally, in order to further investigate how attentional resources are allocated when attention is divided during access, we studied trial-by-trial variability in response errors on the two clocks in the double-cue condition (Figure 3). There was a significant positive correlation for all observers individually, even for the 2 observers without a consistent left-clock bias. When two clocks were cued, response errors on the two clocks covaried $(.15<r<.38$, $M \pm S E M=.24 \pm .02$; all $p$ s $<.022$ ). Figure 3 shows this shared variance. Thus, although it is possible to divide attention in order to access multiple objects or locations, attentional access at these locations remains interdependent. One interpretation is that attentional resources are allocated in parallel to the two locations and that either the two attentional foci share a single access mechanism (e.g., a global "go!" signal) or both depend on the same attentional state (e.g., the observer's level of alertness). In such an account, the consistently lower precision cost for the left clock might be explained by a bias to allocate more attentional resources to the left clock. However, a parallel access interpretation does not explain the difference in time cost for access to the two locations. The leftclock time cost advantage is direct evidence that access to the two locations takes place sequentially, in a consistent order. In this way, attentional access at the first location determines the moment that attentional resources become available for accessing the second location. Note that in this interpretation, postaccess processing at the first location need not necessarily be complete before the second location is accessed, and indeed, attentional resources may well be allocated in parallel to the two streams both before access and during postaccess processing. Our data 


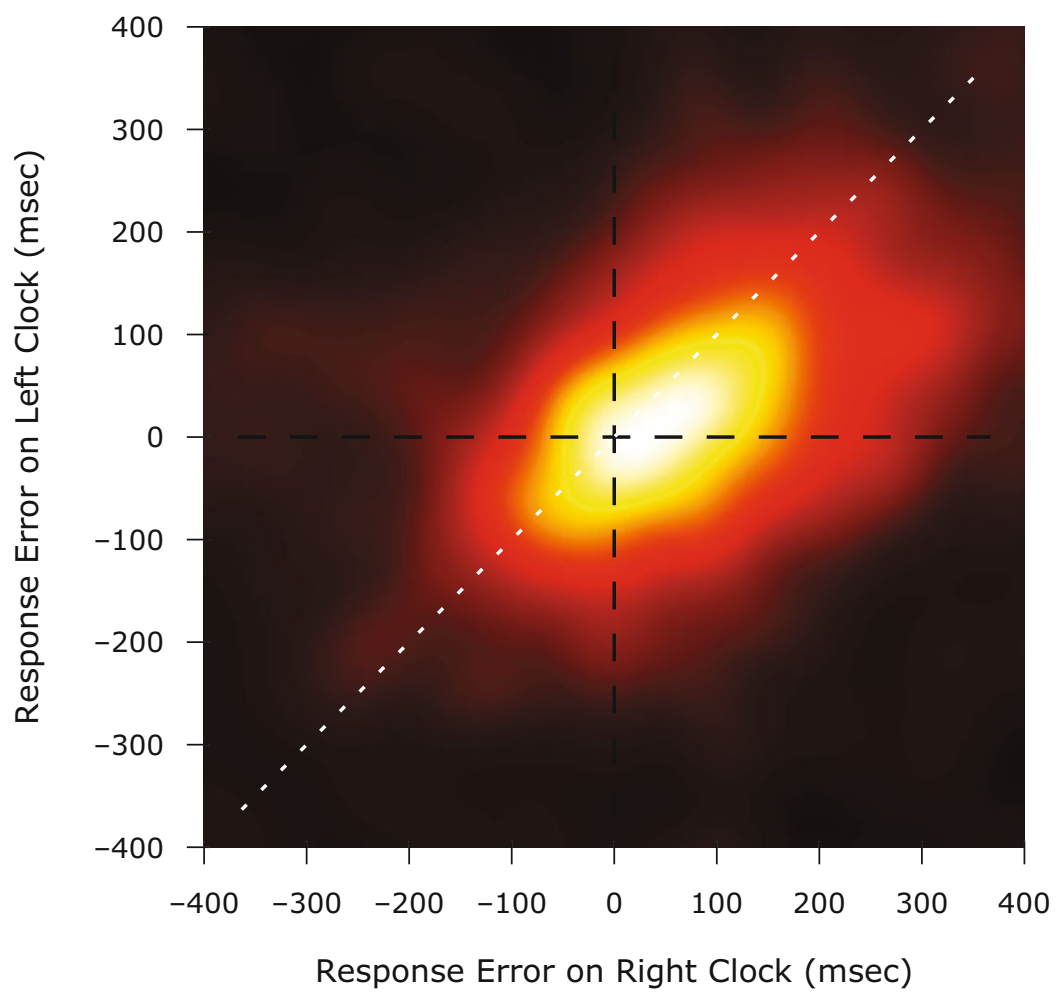

Figure 3. Interdependent access at two locations. A color-coded 2-D histogram (heat map) graphically shows the relationship between response error on left and right clocks for double-cue trials in Experiment 2. There is a clear positive correlation between response error on the left and right clocks. The white dotted line denotes equal response error. The majority of the heat map falls just right of this line, indicating that right-clock response error was consistently higher than left-clock response error.

simply show that access itself takes place at the two locations sequentially.

\section{Eyetracking}

Eye movement recordings for 3 of the 10 observers showed that the observers experienced minimal difficulty maintaining fixation (defined as a circle of $1^{\circ}$ radius surrounding the fixation point) in either experiment. The 3 observers maintained fixation on an average of 353.7 out of 360 trials (98.3\%) in Experiment 1 and on an average of 397.7 out of 400 trials (99.4\%) in Experiment 2. Importantly, fixation was never broken before presentation of the cue; in each instance, a single saccade was made directly to a single target clock in response to the cue. Saccade trials were divided evenly across all conditions in both experiments. There were too few saccade trials to draw meaningful conclusions from observer responses on those trials.

Overall, the results from saccade-free trials for these 3 observers were highly similar to results for the full data set. Although 3 observers provide only limited statistical power, several key effects did reach significance: the progressive time cost as additional clocks were to be monitored in Experiment $1[F(5)=13.69, p<.001]$, the leftclock advantage on time cost $[F(1)=67.63, p=.014]$, and the additional precision cost for reporting two clocks $[F(1)=33.65, p=.029]$ in Experiment 2. The fact that these 3 observers demonstrated the same effects that were evident in the full data set, while maintaining fixation on the vast majority of trials, rules out systematic variations in fixation patterns or eye movements as a possible explanation for the time and precision costs observed in Experiments 1 and 2 .

\section{DISCUSSION}

A general model of divided attention, in which evidence from the wide range of divided attention paradigms can be integrated, is lacking in the literature. An important reason for this is that the stage of processing at which attention is divided (e.g., monitoring or access) and the attentional mechanisms that are affected (optimization and selection) are not always dissociated or made explicit. In order to develop such a model, we investigated the costs of dividing visual attention at two stages of visual processing: monitoring and access. To evaluate the costs of dividing attention both in terms of optimization and in terms of selection, we separately measured time costs and precision costs.

When attention was divided during monitoring (Experiment 1), gradually increasing time costs were observed as the observers monitored additional locations, confirming that it is possible to divide attention into multiple foci and that the availability of attention results in processing optimization at those locations. Division of attention during 
monitoring additionally resulted in a one-off cost to spatiotemporal precision, which is independent of the number of foci into which attention is divided. This incremental precision cost indicates that split foci do not convey the same selection benefit to visual processing that a single, undivided focus does. The constant precision cost when between two and six locations are monitored additionally indicates that during monitoring, attentional resources can be divided over foci in parallel.

When attention was divided during access (Experiment 2), in order to process visual information from two locations, an additional cost to precision was evident at both locations, suggesting that attentional resources available to access visual information is severely limited We cannot fully exclude the possibility that this result is due to the increased memory load of recalling and reporting two hand orientations. Phillips and Christie (1977) argued that visual working memory has a capacity of just a single item. At this point, we cannot distinguish whether the bottleneck is at the level of attentional access (and, therefore, transfer into visual working memory) or at the level of visual working memory itself. One might speculate, however, that there would be no advantage to having greater capacity for attentional access than for storage in short-term memory. An interpretation in terms of attention is furthermore consistent with previous work showing similar limits for the simultaneous attentional access of multiple targets (Duncan, 1980b).

Interestingly, the observers consistently showed lower time costs for left-clock reports than for right-clock reports, on both single-cue and double-cue trials. This suggests that when attention is divided during access, visual information at the divided foci is, in fact, accessed sequentially. In support of this interpretation, we observed that response errors for the two clocks on double-cue trials were correlated within individual observers, exactly as one would predict if access time to the second of two sequentially accessed clocks depended on access time to the first (Figure 3). Note that the choice to consistently allocate attention to the left clock before the right, rather than vice versa, was essentially arbitrary, although we speculate that the observers may have chosen this direction because it is consistent with reading direction. In fact, any trial-by-trial deviation from this strategy would result in underestimation of the benefit associated with attentional priority, and it is therefore likely that the true costs of being the second of the two locations to be accessed by attention are larger than the mean costs observed here.

The finding that different attentional limits constrain the division of attention during monitoring and access is consistent with previous reports (e.g., Duncan, 1980b; Pashler, 1998; Shiffrin et al., 1973). However, the dissociable effects of dividing attention on both processing optimization (in the form of time costs) and selection (in the form of precision costs), at two stages of visual processing, provide a basis on which to build the general model of divided attention that is needed to integrate the wide range of experimental findings evident in the literature. Taken together, our results suggest that divided attention is best understood as the simultaneous preparation and subsequent sequential execution of multiple covert shifts of undivided attention. The gradual increase in time cost observed as additional locations are monitored indicates that several such attentional shifts can be prepared in parallel, although the attentional resources available to do so are limited. This interpretation also explains the time cost asymptote as additional locations are monitored, since the monitoring benefit becomes progressively diluted as additional attentional shifts are prepared. Preparing attentional shifts to more than three or four specific locations ceases to provide any advantage over an exogenously guided attentional shift in response to the cue. The one-off precision cost of dividing attention during monitoring, irrespective of the number of attentional foci into which it is divided, shows that undivided attention provides a unique selection benefit. When only one location is monitored, undivided attention can be preallocated to select the target location before it is cued, whereas when multiple locations are monitored, selection of the target can take place only once it is specified by the cue. Reducing the selected subset from all the monitored clocks to just the target clock introduces additional spatiotemporal variance in observer reports. Finally, the systematically greater time cost of accessing the right clock in Experiment 2 shows that when attention is "divided" during access, the two locations are, in fact, accessed sequentially. The correlation between left- and right-clock reports on double-cue trials provides convergent evidence for this interdependence of access at two locations.

This divided-attention model provides a framework that can integrate diverse findings from the divided-attention literature. When multiple locations are monitored, the attention system prepares shifts of covert attention to these locations. Unitary attention is therefore more rapidly deployed to monitored locations, resulting in performance benefits on masked detection and discrimination tasks at these locations (e.g., Castiello \& Umiltà, 1992). We observed time cost benefits at up to three monitored locations, consistent with limits observed in attentive-tracking paradigms in which attention must similarly be divided across multiple moving objects (e.g., Cavanagh \& Alvarez, 2005; Pylyshyn \& Storm, 1988). Although some degree of visual processing must already take place at the monitoring stage, since the appearance of a potential target must be detected and the motion speed and direction of a tracked object must be updated, attentional access is required for a detailed, explicit representation to become available to consciousness. This is consistent with previous accounts of attentive-tracking mechanisms (e.g., fINST's; Trick \& Pylyshyn, 1989) and more recent attentive-tracking results that show that observers fail to notice feature changes in objects they are tracking (Bahrami, 2003). Finally, because the monitored regions are simply potential shift targets, they need not necessarily be contiguous (e.g., Awh \& Pashler, 2000; Kramer \& Hahn, 1995).

Although attention can monitor multiple locations in parallel, in this model, attention cannot access information from these locations simultaneously. Instead, the attention system executes sequential, planned shifts of undivided 
attention to these locations. Access, in this context, refers to specific attentional sampling of visual information at a particular point in space and time, with maximum spatiotemporal precision. Clearly, not all the visual information of which we become conscious is accessed in this sense, in the same way that we can consciously perceive a scene without being conscious of a changing detail in that scene unless it is attended (Rensink et al., 1997). In addition, although data from the present study support the serial allocation of attention during access, this does not rule out the theoretical possibility that simultaneous parallel access of separate locations could occur under less stringent perceptual limits. Because, in Experiment 2, the observers were required to maintain the identity of the two accessed clocks, they may have adopted a serial access strategy in order to avoid confusing the two clocks. One might also argue that the dual-task condition in Experiment 2 is in itself better suited to detecting a possible serial mechanism than is the single-task situation in Experiment 1. However, note that the results of Experiment 1 were inconsistent with the specific consequences of serial monitoring and that, even in Experiment 2, no effect would have been observed if the observers had not all chosen to favor the left clock over the right. The precise degree to which visual information requires attentional access (and, therefore, serial allocation of attentional resources) to become available to consciousness, and under which conditions attention might be able to access multiple locations truly simultaneously, therefore remain questions for future study (for a set of proposed constraints, see Huang \& Pashler, 2007).

Furthermore, this interpretation of divided attention is in line with considerable evidence implicating attention in the preparation of overt, rather than covert, shifts of attention: saccadic eye movements (e.g., Castet, Jeanjean, Montagnini, Laugier, \& Masson, 2006; Filali-Sadouk, Castet, Olivier, \& Zenon, 2010; Hunt \& Cavanagh, 2009; Kowler, Anderson, Dosher, \& Blaser, 1995; Montagnini \& Castet, 2007; Rizzolatti, Riggio, Dascola, \& Umiltà, 1987). Strikingly, it has recently been shown that when two (Godijn \& Theeuwes, 2003) or even three (Baldauf \& Deubel, 2008) successive saccades are prepared, enhanced processing is observed at each of the saccade targets, well before execution of the saccade. In our experiments, we similarly observed processing benefits at up to four monitored locations. However, Deubel (2008) has reported that processing performance increases sharply at the saccade target location immediately prior to execution of the saccade, accompanied by disengagement of attention at the other locations. Although potential saccade targets, therefore, do benefit from divided attention as the saccade is prepared, actual execution of the saccade involves the sequential allocation of undivided attention. This is consistent with our finding that although attention can be divided to monitor multiple locations, attentional access, in fact, requires undivided attention, even when information is to be selected from multiple locations.

The depth of processing that can be realized by divided attention at each stage of processing remains an important open question, which is particularly relevant in the context of visual search. There does not seem to be a categorical distinction between parallel and serial search behavior (Wolfe, 1998), whereas that distinction is explicit in our model: Dividing attention during monitoring can be done in parallel, whereas multiple locations can be accessed only sequentially. On the whole, parallel and serial mechanisms are notoriously difficult to distinguish (Townsend, 1972). The question is to what degree distractors in a visual search task are actually accessed, in the sense that they are each selectively made available to consciousness. In terms of neural economy, it would seem more logical to presume the existence of a cognitive mechanism that frees up attentional resources tied up in identifying a possible target the moment it is established that it is a distractor, rather than waiting until that information is brought all the way to consciousness. More generally, this might suggest a continuum in terms of depth of processing, ranging from what is processed rapidly and automatically, such as the gist of a scene (Oliva, 2005) or the presence or absence of simple features, at one end, through visual properties that require progressive attentional resources, to those that require full, undivided attention or even foveation. However, on the basis of the present data, such a mechanism remains firmly in the realm of speculation. The degree to which the division of attention is under cognitive control, the overall time course of the different attentional subsystems, and the degree to which they are neurally dissociable are therefore topics for further investigation. We hope that the model we present here, in which the division of attention at different levels of visual processing is understood as the preparation and subsequent execution of covert shifts of undivided attention, will provide an overarching framework within which the stages and mechanisms involved in dividing attention are made explicit, allowing the integration of results from a wide range of past and future experimental paradigms.

\section{AUTHOR NOTE}

This research was supported by a Pioneer grant from the Netherlands Organisation for Scientific Research (NWO) to F.A.J.V. We thank Marjolein Kammers and three anonymous reviewers for their very helpful comments on earlier versions of the manuscript. Correspondence concerning this article should be addressed to H. Hogendoorn, Helmholtz Institute, Experimental Psychology Division, Utrecht University, Heidelberglaan 2, 3584 CS Utrecht, The Netherlands (e-mail: j.h.a.hogendoorn@uu.nl).

\section{REFERENCES}

Alvarez, G. A., \& Cavanagh, P. (2005). Independent resources for attentional tracking in the left and right visual hemifields. Psychological Science, 16, 637-643.

Awh, E., \& Pashler, H. (2000). Evidence for split attentional foci. Journal of Experimental Psychology: Human Perception \& Performance, 26, 834-846.

BAHRAMI, B. (2003). Object property encoding and change blindness in multiple object tracking. Visual Cognition, 10, 949-963.

Baldauf, D., \& Deubel, H. (2008). Properties of attentional selection during the preparation of sequential saccades. Experimental Brain Research, 184, 411-425.

Bichot, N. P., CAVE, K. R., \& Pashler, H. (1999). Visual selection mediated by location: Feature-based selection of noncontiguous locations. Perception \& Psychophysics, 61, 403-423.

Brainard, D. H. (1997). The Psychophysics Toolbox. Spatial Vision, 10, $433-436$. 
Broadbent, D. (1958). Perception and communication. London: Pergamon.

Carlson, T. A., Hogendoorn, H., \& Verstraten, F. A. J. (2006). The speed of visual attention: What time is it? Journal of Vision, 6, 14061411.

Castet, E., Jeanjean, S., Montagnini, A., Laugier, D., \& Masson, G. S. (2006). Dynamics of attentional deployment during saccadic programming. Journal of Vision, 6, 196-212.

Castiello, U., \& Umiltà, C. (1992). Splitting focal attention. Journal of Experimental Psychology: Human Perception \& Performance, 18, 837-848.

Cavanagh, P. (1992). Attention-based motion perception. Science, 257, $1563-1565$.

Cavanagh, P., \& Alvarez, A. (2005). Tracking multiple targets with multifocal attention. Trends in Cognitive Sciences, 9, 349-354.

Deubel, H. (2008). The time course of presaccadic attention shifts. Psychological Research, 72, 630-640.

Duncan, J. (1980a). The demonstration of capacity limitation. Cognitive Psychology, 12, 75-96.

Duncan, J. (1980b). The locus of interference in the perception of simultaneous stimuli. Psychological Review, 87, 272-300.

Filali-Sadouk, N., Castet, E., Olivier, E., \& Zenon, A. (2010). Similar effect of cueing conditions on attentional and saccadic temporal dynamics. Journal of Vision, 10(4, Art. 21), 1-13.

Gobell, J. L., Tseng, C. H., \& Sperling, G. (2004). The spatial distribution of visual attention. Vision Research, 44, 1273-1296.

Godis, R., \& Theeuwes, J. (2003). Parallel allocation of attention prior to the execution of saccade sequences. Journal of Experimental Psychology: Human Perception \& Performance, 29, 882-896.

Hogendoorn, H., Carlson, T. A., \& Verstraten, F. A. J. (2007). The time course of attentive tracking. Journal of Vision, 7(14, Art. 2), $1-10$.

Huang, L., \& Pashler, H. (2007). A Boolean map theory of visual attention. Psychological Review, 114, 599-631.

Hunt, A. R., \& CAVAnagh, P. (2009). Looking ahead: The perceived direction of gaze shifts before the eyes move. Journal of Vision, 9(9, Art. 1), 1-7.

Intriligator, J., \& CAVAnagh, P. (2001). The spatial resolution of visual attention. Cognitive Psychology, 43, 171-216.

Kowler, E., Anderson, E., Dosher, B., \& Blaser, E. (1995). The role of attention in the programming of saccades. Vision Research, 35, 1897-1916.

Kramer, A. F., \& Hahn, S. (1995). Splitting the beam: Distribution of attention over noncontiguous regions of the visual field. Psychological Science, 6, 381-386.

LiBet, B. (2004). Mind time: The temporal factor in consciousness. Cambridge, MA: Harvard University Press.

McCormick, P. A., Klein, R. M., \& Johnston, S. (1998). Splitting versus sharing focal attention: Comment on Castiello and Umiltà (1992). Journal of Experimental Psychology: Human Perception \& Performance, 24, 350-357.

McMains, S. A., \& Somers, D. C. (2004). Multiple spotlights of attentional selection in human visual cortex. Neuron, 42, 677-686.

Montagnini, A., \& CAstet, E. (2007). Spatiotemporal dynamics of visual attention during saccade preparation: Independence and coupling between attention and movement planning. Journal of Vision, 7(14, Art. 8), 1-16.

Oliva, A. (2005). Gist of the scene. In L. Itti, G. Rees, \& J. K. Tsotsos (Eds.), The encyclopedia of the neurobiology of attention (pp. 251256). Amsterdam: Elsevier.

PALMER, J. (1995). Attention in visual search: Distinguishing four causes of a set-size effect. Current Directions in Psychological Science, $\mathbf{4}$, 118-123.

PASHLER, H. (1998). The psychology of attention. Cambridge, MA: MIT Press.

Pelli, D. G. (1997). The VideoToolbox software for visual psychophysics: Transforming numbers into movies. Spatial Vision, 10, 437-442.

Phillips, W. A., \& Christie, D. F. M. (1977). Components of visual memory. Quarterly Journal of Experimental Psychology, 29, 117-133.

Posner, M. I. (1980). Orienting of attention. Quarterly Journal of Experimental Psychology, 32, 3-25.

PyLYShyn, Z. W., \& STORM, R. W. (1988). Tracking multiple independent targets: Evidence for a parallel tracking mechanism. Spatial Vision, 3, 179-197.

Rensink, R. A., O’Regan, J. K., \& Clark, J. J. (1997). To see or not to see: The need for attention to perceive changes in scenes. Psychological Science, 8, 368-373.

Rizzolatti, G., Riggio, L., Dascola, I., \& Umiltà, C. (1987). Reorienting attention across the horizontal and vertical meridians: Evidence in favor of a premotor theory of attention. Neuropsychologia, 25, 31-40.

Scholl, B. J., Pylyshyn, Z. W., \& Feldman, J. (2001). What is a visual object? Evidence from target merging in multiple object tracking. Cognition, 80, 159-177.

Shiffrin, R. M., Gardner, G. T., \& Allmeyer, D. H. (1973). On the degree of attention and capacity limitations in visual processing. Perception \& Psychophysics, 14, 231-236.

TownSEND, J. T. (1972). Some results concerning the identifiability of parallel and serial processes. British Journal of Mathematical \& Statistical Psychology, 25, 168-199.

Treisman, A. M., \& Gelade, G. (1980). A feature integration theory of attention. Cognitive Psychology, 12, 97-136.

Trick, L., \& Pylyshyn, Z. W. (1989). Subitizing and the FINST spatial index model [Abstract]. Bulletin of the Psychonomic Society, 27, 490.

VanRullen, R., Carlson, T. A., \& Cavanagh, P. (2007). The blinking spotlight of attention. Proceedings of the National Academy of Sciences, 104, 19204-19209.

Wolfe, J. M. (1994). Guided Search 2.0: A revised model of visual search. Psychonomic Bulletin \& Review, 1, 202-238.

Wolfe, J. M. (1998). What can 1 million trials tell us about visual search? Psychological Science, 9, 33-39.

WundT, W. M. (1883). Philosophische studien. Leipzig: Engelmann.

YANTIS, S. (1992). Multielement visual tracking: Attention and perceptual organization. Cognitive Psychology, 24, 295-340.

\section{NOTE}

1. The monitor 2 /access 1 condition was tested in both experiments, with comparable estimates of precision cost but a substantial $(\sim 50 \mathrm{msec})$ difference in time cost. One possible explanation for this apparent discrepancy is the presence of distractor clocks in Experiment 1. Although in visual search tasks, monitoring two out of six locations has been found to be equivalent to monitoring two out of two locations (Palmer, 1995), it is unknown to what degree that result generalizes to the dynamic stimuli used in the clocks task. Alternatively, the discrepancy could be the result of strategy (because, in Experiment 2, the observers were prepared for the possibility of two simultaneous targets) or repetition (in Experiment 2 , the same two clocks were monitored throughout the experiment, whereas in Experiment 1, a different subset of one to six clocks was monitored on each trial).

(Manuscript received July 6, 2009; revision accepted for publication June 4, 2010.) 